\title{
Effect of a uniform bias force on the Brownian movement in double-well potentials
}

\author{
W. T. Coffey ${ }^{*}$ \\ Department of Electronic and Electrical Engineering, Trinity College, Dublin 2, Ireland \\ D. S. F. Crothers \\ Department of Applied Mathematics and Theoretical Physics, The Queen's University of Belfast, Belfast BT7 1NN, Northern Ireland \\ Yu. P. Kalmykov \\ Centre d'Études Fondamentales, Université de Perpignan, 52 Avenue de Villeneuve, 66860 Perpignan Cedex, France \\ and Institute of Radio Engineering and Electronics of the Russian Academy of Sciences, \\ Vvedenskii Square 1, Fryazino, Moscow Region, 141120, Russian Federation
}

(Received 30 September 1996)

\begin{abstract}
A general property of the Brownian motion in a bistable potential with the asymmetry arising from the imposition of a bias force is described. The property is that, at values of the bias force far less than the critical value required to destroy the bistable nature of the potential, the relaxation process may come to be dominated by fast intrawell modes rather than the slow overbarrier relaxation mode. The effect manifests itself in the pronounced departure of the inverse of the smallest eigenvalue of the Fokker-Planck equation from the relaxation time. The phenomenon is illustrated by two examples. [S1063-651X(97)11804-9]

PACS number(s): 05.40.+j, 05.60+w, 76.20.+q
\end{abstract}

\section{INTRODUCTION}

The Brownian motion in bistable potentials is a crucial factor in the thermally activated switching behavior of systems which may exist in either of two stable states. We mention $[1,2]$ a chemical reaction where two distinct chemical species are separated by a (internal) potential barrier; the reaction being modeled $[1,2]$ by thermally activated diffusion over the barrier. Another example is a fine single domain ferromagnetic particle with uniaxial anisotropy so possessing two stable magnetic states $[2,3,6]$. Orientation of the magnetization of such a particle may undergo a reversal (i.e., cross the anisotropy internal potential barrier) due to thermal agitation. The stochastic dynamics of the chemical problem in the high damping (noninertial) limit will be governed by the Smoluchowski equation in the reaction coordinates $[1,4,5]$. In the magnetic problem the inertia of the particle plays no role; thus the relaxation is governed by a FokkerPlanck equation (FPE) in the space of orientations of the magnetization only.

In both problems the time to cross the potential barrier (mean first passage time) in the high barrier (low temperature) limit may be obtained from the inverse of the smallest nonvanishing eigenvalue $\lambda_{1}$ of the Sturm-Liouville equation associated with the appropriate FPE. Thus the barrier crossing process in the high barrier limit is described by a single eigenmode of the FPE.

Yet another quantity describing the relaxation process is $[1,3]$ the correlation time $\tau$ which is defined as the area under the curve of the normalized autocorrelation function (ACF) $C(t)$ of the appropriate dynamic quantity. The correlation time, which is a global characterization of the relaxation pro-

*Corresponding author. cess involved, contains contributions from all the eigenvalues of the FPE and is related to the escape rate over the potential barrier. In the magnetic problem $[3,6] \tau$ is the correlation time of the normalized equilibrium $\mathrm{ACF}$ of the magnetization of the particle. In the chemical reaction problem $[4,5] \tau$ is the correlation time of the normalized ACF of the reaction coordinate (position). The asymptotic behavior of $\tau$ is often similar to $\lambda_{1}^{-1}$ showing that the relaxation process is dominated by the "long lived" barrier crossing mode. Marked differences occur, however, in the magnetic problem if a strong external uniform bias field is applied along the anisotropy axis. Here $\tau$ will diverge exponentially from $\lambda_{1}^{-1}$ in the high anisotropy (low temperature) limit $[3,6]$. Moreover, such behavior occurs at a critical value $h_{c}$ of the ratio $h$, i.e., bias field parameter-anisotropy barrier height parameter, far less than the value needed to destroy the bistable nature of the potential. Thus in the low temperature limit the relaxation process is no longer dominated by the slow decay mode associated with the barrier crossing at values of $h$ in excess of the critical value. The phenomenon was first noted for the magnetic problem in Ref. [6] and later explained by Garanin [3]. He showed that it is a natural consequence of the depletion of the shallower of the two potential wells by the uniform field. Thus at low temperatures the fast modes in the deeper well may come to dominate the relaxation.

In this paper we prove that the uniform bias force effect always exists in relaxation in one-dimensional bistable potentials and we assert that such an effect is a general feature of relaxation in biased double-well potentials. We bolster our conclusions by illustrating the effect for the two disparate examples of relaxation in a biased 2-4 potential and orientational relaxation in a biased uniaxial anisotropy potential given below. 


\section{RELAXATIONAL DYNAMICS OF A BROWNIAN PARTICLE IN A BIASED DOUBLE-WELL POTENTIAL}

The one-dimensional noninertial translational Brownian motion of a particle in a double-well potential $V(x)$ is governed by the noninertial Fokker-Planck (Smoluchowski) equation for the probability distribution function $W$ of the position which is [1]

$$
\zeta \frac{\partial}{\partial t} W=\frac{\partial}{\partial x}\left(W \frac{\partial}{\partial x} V\right)+k T \frac{\partial^{2}}{\partial x^{2}} W
$$

where $\zeta$ is the friction coefficient, $k$ is the Boltzmann constant, and $T$ is the absolute temperature.

The quantities of interest are the positional ACF

$$
C(t)=\langle x(0) x(t)\rangle_{0}-\langle x(0)\rangle_{0}^{2},
$$

where the symbol \langle\rangle$_{0}$ designates the equilibrium ensemble average, and the correlation time

$$
\tau=\frac{1}{C(0)} \int_{0}^{\infty} C(t) d t
$$

A general equation for the correlation time $\tau$ for a system governed by Eq. (1) has been given by Risken (Ref. [1], Eq. (S9.14))

$$
\begin{aligned}
\tau= & \frac{\beta \zeta}{Z\left[\left\langle x^{2}\right\rangle_{0}-\langle x\rangle_{0}\right]} \int_{-\infty}^{\infty} e^{\beta V(x)} \\
& \times\left[\int_{-\infty}^{x}\left(x^{\prime}-\langle x\rangle_{0}\right) e^{-\beta V\left(x^{\prime}\right)} d x^{\prime}\right]^{2} d x,
\end{aligned}
$$

where

$$
\begin{gathered}
Z=\int_{-\infty}^{\infty} e^{-\beta V(x)} d x, \quad\langle x\rangle_{0}=\frac{1}{Z} \int_{-\infty}^{\infty} x e^{-\beta V(x)} d x, \\
\left\langle x^{2}\right\rangle_{0}=\frac{1}{Z} \int_{-\infty}^{\infty} x^{2} e^{-\beta V(x)} d x
\end{gathered}
$$

and $\beta=(k T)^{-1}$.

For the purpose of our discussion we consider a biased double-well potential

$$
\beta V(x)=\sigma[U(x)-h x]
$$

with a maximum at $x_{B}$ and minima at $x_{A}$ and $x_{C}$ and without loss of generality we let $V\left(x_{C}\right)>V\left(x_{A}\right)$ (see Fig. 1).

At low temperatures $(\sigma \gg 1) C(t)$ may be approximated by two exponentials

$$
C(t) \approx \Delta_{\mathrm{well}} e^{-t / \tau_{\mathrm{well}}}+\Delta_{B} e^{-t \lambda_{1}},
$$

where $\tau_{\text {well }}$ is the relaxation time in the deep well, and so has a weak temperature dependence, $\lambda_{1}$ is the smallest eigenvalue of the Sturm-Liouville equation associated with the FPE and so has Arrhenius (exponential) temperature dependence, and correspondingly $\Delta_{\text {well }} \gg \Delta_{B}$. These two exponentials correspond to intrawell and overbarrier relaxation processes. According to Eq. (3)

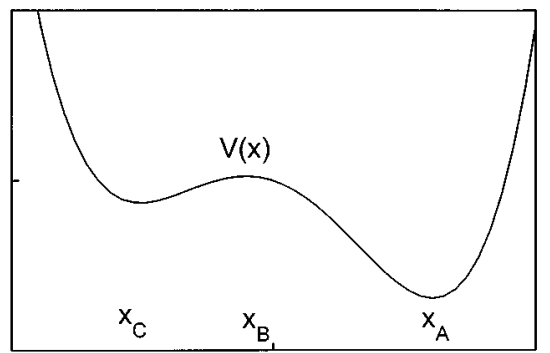

FIG. 1. Double-well potential with local minima at $x_{A}$ and $x_{C}$ and a local maximum at $x_{B}$.

$$
\tau \approx \frac{\tau_{\mathrm{well}} \Delta_{\mathrm{well}}+\Delta_{B} \lambda_{1}^{-1}}{\Delta_{\mathrm{well}}+\Delta_{B}} \approx \tau_{\mathrm{well}}+\Delta_{B} \lambda_{1}^{-1} / \Delta_{\mathrm{well}}
$$

Recognizing that the partition function $Z$ is the sum of the contributions due to the two potential wells and applying the steepest descent method to the evaluation of $Z$, we obtain

$$
\begin{aligned}
\frac{\Delta_{B}}{\Delta_{\text {well }}} \sim & \frac{e^{-\sigma\left[U\left(x_{C}\right)-U\left(x_{A}\right)-h\left(x_{A}-x_{C}\right)\right]}}{(1-2 / \pi)}\left(\frac{U^{\prime \prime}\left(x_{A}\right)}{U^{\prime \prime}\left(x_{C}\right)}\right)^{1 / 2} \\
& \times\left[\frac{4}{\pi}-1+\frac{4}{\pi}\left(\frac{U^{\prime \prime}\left(x_{A}\right)}{U^{\prime \prime}\left(x_{C}\right)}\right)^{1 / 2}+\frac{U^{\prime \prime}\left(x_{A}\right)}{U^{\prime \prime}\left(x_{C}\right)}\right] .
\end{aligned}
$$

According to the Kramers' escape rate theory the inverse of the smallest nonvanishing eigenvalue has exponential dependence on the barrier height [1], viz.,

$$
\lambda_{1}^{-1} \sim e^{\sigma\left[U\left(x_{B}\right)-U\left(x_{C}\right)+h\left(x_{C}-x_{B}\right)\right]} .
$$

Thus

$$
\Delta_{B} \lambda_{1}^{-1} / \Delta_{\text {well }} \sim e^{\sigma\left[U\left(x_{B}\right)+U\left(x_{A}\right)-2 U\left(x_{C}\right)+h\left(2 x_{C}-x_{B}-x_{A}\right)\right]} .
$$

Now the argument of the exponential, viz.,

$$
\sigma\left[U\left(x_{B}\right)+U\left(x_{A}\right)-2 U\left(x_{C}\right)+h\left(2 x_{C}-x_{B}-x_{A}\right)\right],
$$

may change its sign at some critical value of $h_{c}$. This is the crucial fact underpinning all that follows. Thus if $h<h_{c}$ the quantity $\Delta_{B} \lambda_{1}^{-1} / \Delta_{\text {well }}$ increases exponentially as the temperature $T$ decreases and so completely determines the temperature dependence of the correlation time $\tau$. If $h>h_{c}$, on the other hand, the quantity $\Delta_{B} \lambda_{1}^{-1} / \Delta_{\text {well }}$ decreases exponentially as $T$ decreases, thus $\tau$ no longer has Arrhenius behavior. At this critical value of the bias parameter the relaxation switches from being dominated by the behavior of the smallest nonvanishing eigenvalue $\lambda_{1}$ to being dominated by the fast relaxation processes in the deep well of the potential because of the depletion of the upper (shallow) well at low temperatures [3]. We shall now demonstrate that this behavior occurs at values of $h$ such that the double-well structure of the potential still persists.

As a definite example consider the double-well potential

$$
\beta V(x)=\frac{\sigma}{4}\left[x^{4}-4 x^{2}-h x\right]
$$




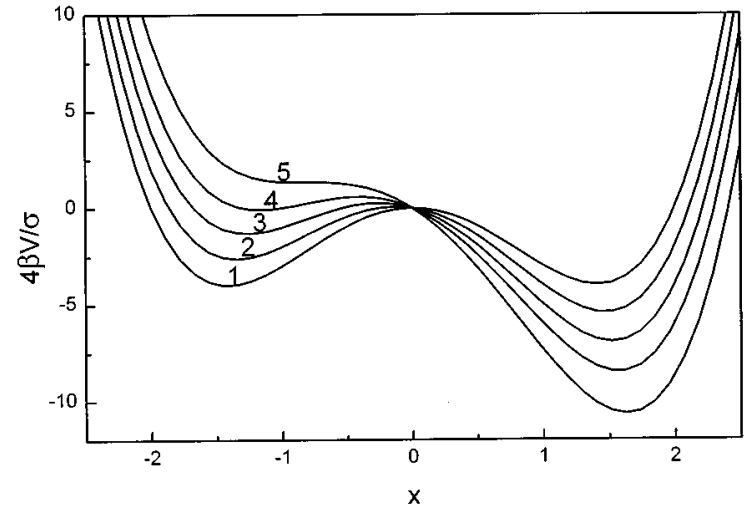

FIG. 2. Potential $4 \beta V(x) / \sigma=-h x-4 x^{2}+x^{4}$ from Eq. (13) as a function of $x$ for various values of the bias parameter $h$ : 0 (curve 1), 1 (curve 2), 2 (curve 3), 3 (curve 4), and $16 \sqrt{2 / 27}$ (curve 5).

where $x$ is a dimensionless coordinate and $h$ is the bias parameter. At $h=0$ the potential Eq. (13) is symmetrical and has a barrier at $x=0$ where the potential has a maximum with height relative to the minimum equal to $\sigma$ (in dimensionless units).

The relaxational dynamics in this case are well known (see, e.g., Refs. [4,5]) and may be described as follows. The correlation time for all barrier height parameters is given by Eq. (4) with $\langle x\rangle_{0}=0$ [4]. In the low temperature (high barrier) limit the ACF may be approximated by $[4,5]$

$$
C(t) \approx \Delta_{\text {well }} e^{-t / \tau_{\text {well }}+\Delta_{B} e^{-t \lambda_{1}},}
$$

where

$$
\begin{gathered}
\tau_{\text {well }}=\beta \zeta\left(\left\langle x^{2}\right\rangle_{0}-\langle x\rangle_{\text {well }}^{2}\right), \quad \lambda_{1}^{-1} \approx \frac{\pi \beta \zeta\left\langle x^{2}\right\rangle_{0} e^{\sigma}}{4 \sqrt{2} \sigma}, \\
\Delta_{\text {well }} \approx\langle x\rangle_{\text {well }}^{2}, \quad \Delta_{B} \approx\left\langle x^{2}\right\rangle_{0}-\langle x\rangle_{\text {well }}^{2},
\end{gathered}
$$

and $\langle x\rangle_{\text {well }}$ is the average of $x$ in one of the wells (e.g., over the range $0 \leqslant x<\infty)$. Thus $\tau$ has the pronounced Arrhenius behavior

$$
\tau \approx \lambda_{1}^{-1} \frac{\Delta_{B}}{\Delta_{\text {well }}} \sim e^{\sigma}
$$

For $h \neq 0$ the potential becomes asymmetrical (see Fig. 2). Moreover, the double-well structure of the potential disappears at

$$
h=h_{s}=16 \sqrt{2 / 27} \approx 4.35465 .
$$

Now the correlation time is also given by the exact Eq. (4). The results of the exact calculation of $\tau$ are shown in Fig. 3 . It is apparent that $\tau$ ceases to have Arrhenius behavior at values of $h$ that are considerably smaller than the critical parameter $h_{s}$ of Eq. (18). Furthermore, one can see in Fig. 3 that for a given shape of the potential $(h=$ const) the behavior of $\tau$ may dramatically alter from exponentially increasing to exponentially decreasing (see curve 4). Applying the approximate treatment of Sec. II by means of Eq. (11) we see that

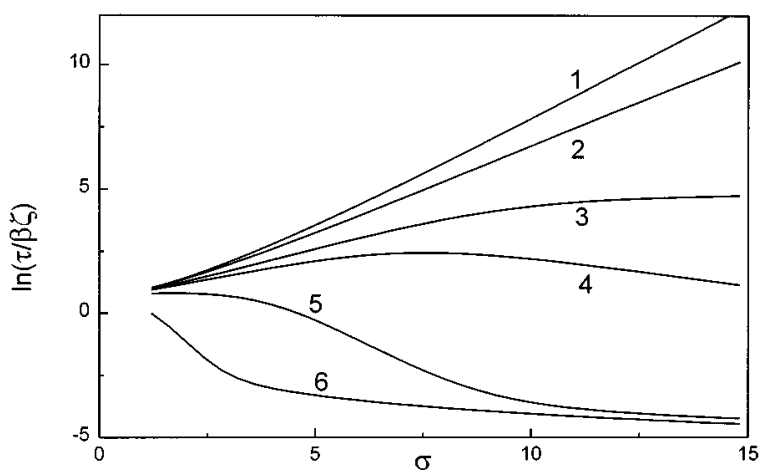

FIG. 3. $\ln (\tau / \beta \zeta)$ calculated from Eqs. (4) and (13) as a function of $\sigma$ for various values of $h$ : 0 (curve 1), 0.5 (curve 2), 0.95 (curve 3), 1.2 (curve 4), 2 (curve 5), and 4.5 (curve 6).

$$
\lambda_{1}^{-1} \frac{\Delta_{B}}{\Delta_{\text {well }}} \sim e^{\sigma[1-(3 / 2 \sqrt{2}) h]}
$$

alters its behavior from exponential increase to exponential decrease at $h_{c} \approx 2 \sqrt{2} / 3 \approx 0.943$.

\section{ORIENTATIONAL RELAXATION IN A BIASED POTENTIAL}

The FPE for the probability distribution function $W$ of the orientations of magnetization of an assembly of single domain ferromagnetic particles is [2]

$$
\begin{aligned}
2 \tau_{0} \frac{\partial}{\partial t} W= & \frac{\beta}{\sin \vartheta} \frac{\partial}{\partial \vartheta}\left((\sin \vartheta) W \frac{\partial}{\partial \vartheta} V\right) \\
& +\frac{1}{\sin \vartheta} \frac{\partial}{\partial \vartheta}\left((\sin \vartheta) \frac{\partial}{\partial \vartheta} W\right)
\end{aligned}
$$

where $\tau_{0}$ is a characteristic (Néel) relaxation time. We note that the FPE (20) is similar to the noninertial Smoluchowski equation for the probability distribution function $W$ of the orientations of a polar molecule in nematic liquid crystals in the mean field approximation [8]

In applications of linear response theory to dielectric and magnetic relaxation the quantity of interest is the dipole moment equilibrium $\mathrm{ACF}$ [2]

$$
C(t)=\langle(\cos \vartheta)(0)(\cos \vartheta)(t)\rangle_{0}-\langle(\cos \vartheta)(0)\rangle_{0}^{2},
$$

which describes the step-off linear response of polarization and magnetization. The correlation time $\tau$ for an arbitrary axially symmetrical potential $V$ is $[3,7]$

$$
\begin{aligned}
\tau= & \frac{2 \tau_{0}}{Z\left[\left\langle\cos ^{2} \vartheta\right\rangle_{0}-\langle\cos \vartheta\rangle_{0}^{2}\right]} \int_{-1}^{1} e^{\beta V(z)} \\
& \times\left[\int_{-1}^{z}\left(z^{\prime}-\langle\cos \vartheta\rangle_{0}\right) e^{-\beta V\left(z^{\prime}\right)} d z^{\prime}\right]^{2} \frac{d z}{1-z^{2}}
\end{aligned}
$$

where 


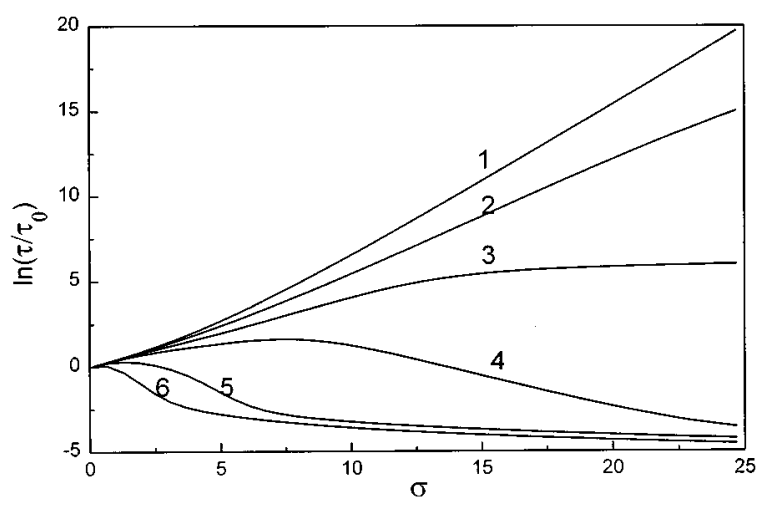

FIG. 4. $\ln \left(\tau / \tau_{0}\right)$ calculated from Eqs. (22) and (24) as a function of $\sigma$ for different values of $h$ : 0 (curve 1), 0.1 (curve 2), 0.17 (curve 3), 0.25 (curve 4), 0.5 (curve 5), and 1 (curve 6).

$$
\begin{gathered}
Z=\int_{-1}^{1} e^{-\beta V(z)} d z, \quad\langle\cos \vartheta\rangle_{0}=\frac{1}{Z} \int_{-1}^{1} z e^{-\beta V(z)} d z \\
\left\langle\cos ^{2} \vartheta\right\rangle_{0}=\frac{1}{Z} \int_{-1}^{1} z^{2} e^{-\beta V(z)} d z
\end{gathered}
$$

As an example consider the simplest uniaxial potential

$$
\beta V(z)=-\sigma\left(z^{2}+2 h z\right) \quad(z=\cos \vartheta),
$$

where $\sigma$ and $h$ are the dimensionless anisotropy and external field parameters, respectively. At $h=0$ the potential Eq. (24) is symmetrical and has a barrier at $\vartheta=\pi / 2$, where the potential has a maximum where the height relative to the minima at $\vartheta=0$ and $\vartheta=\pi$ is equal to $\sigma$. For $h \neq 0$ the potential becomes asymmetrical and the double-well structure disappears at $h=h_{s}=1$.

The relaxational dynamics have been investigated in detail in Refs. [6,3] and may be described as follows. The correlation time $\tau$ is given by the exact Eq. (22). The results of the exact calculation of $\tau$ are shown in Fig. 4. It is apparent that $\tau$ ceases to display Arrhenius behavior at values of $h$ that are considerably smaller than the critical value $h_{s}$, i.e., when the double-well structure of the potential is still present. In the low temperature limit the ACF may be approximated by [3]

$$
C(t) \approx \Delta_{\mathrm{well}} e^{-t / \tau_{\mathrm{well}}}+\Delta_{B} e^{-t \lambda_{1}},
$$

where

$$
\begin{gathered}
\tau_{\mathrm{well}} \approx \frac{\tau_{0}}{2 \sigma(1+h)}, \\
\lambda_{1}^{-1} \approx \tau_{0} \pi^{1 / 2} \sigma^{-3 / 2}(1+3 h) e^{\sigma(1-2 h)}+O\left(h^{2}\right), \\
\Delta_{B} / \Delta_{\mathrm{well}} \approx 16 \sigma^{2} e^{-4 \sigma h}+O\left(h^{2}\right) .
\end{gathered}
$$

Thus

$$
\lambda_{1}^{-1} \frac{\Delta_{B}}{\Delta_{\text {well }}} \sim e^{\sigma(1-6 h)}
$$

alters its behavior from exponential increase to exponential decrease at $h_{c} \approx 1 / 6 \approx 0.16667$ [3], which is again considerably smaller than $h_{s}$. This is in agreement with the originally reported value of $h_{c}$ [6].

The above results may be used for the evaluation of the linear response of diverse physical systems. In particular, they can be applied with a small modification to the calculation of the linear dielectric response of nematic liquid crystals and systems of polar and polarizable molecules in a strong dc bias field and to the corresponding magnetic response of an assembly of single domain ferromagnetic particles. In all cases the longitudinal relaxation (dielectric and magnetic, respectively) of these systems is governed by the FPE with the uniaxial potential given by Eq. (24) and appropriate interpretation of the parameters $\tau_{0}, h$, and $\sigma$ (for details see Refs. [8-12]). Finally we remark that (on account of the linear response theory) the bias field effect is of particular significance for the linear alternating current (ac) response of particles in a bistable potential. It appears that even for a very small change in $h$ near the critical value of $h_{c}$ that there will be a marked change in the ac response as the relaxation switches from being dominated by the low-frequency barrier crossing mode to the high-frequency modes associated with the intrawell relaxation (e.g., Ref. [6]).

\section{ACKNOWLEDGMENTS}

One of us (W.T.C.) acknowledges the financial support of the Forbairt Research Collaboration Fund and the French Foreign Office. Y.P.K. thanks the French Ministry of High Education and Research for high level Grant No. PECO-CEI. The partial support of this work by the Russian Foundation for Basic Research (Grant No. 96-02-16762-a) is also gratefully acknowledged.
[1] H. Risken, The Fokker-Planck Equation, 2nd ed. (Springer, Berlin, 1989).

[2] W. T. Coffey, Yu. P. Kalmykov, and J. T. Waldron, The Langevin Equation (World Scientific, Singapore, 1996).

[3] D. A. Garanin, Phys. Rev. E 54, 3250 (1996).

[4] A. Perico, R. Pratolongo, K. F. Freed, R. W. Pastor, and A. Szabo, J. Chem. Phys. 98, 564 (1993).

[5] Yu. P. Kalmykov, W. T. Coffey, and J. Waldron, J. Chem. Phys. 105, 2112 (1996).

[6] W. T. Coffey, D. S. F. Crothers, Yu. P. Kalmykov, and J. T. Waldron, Phys. Rev. B 51, 15947 (1995).
[7] W. T. Coffey and D. S. F. Crothers, Phys. Rev. E 54, 4768 (1996).

[8] A. J. Martin, G. Meier, and A. Saupe, Symp. Faraday Soc. 5, 119 (1971)

[9] W. F. Brown, Jr., Phys. Rev. 130, 1677 (1963).

[10] Yu. L. Raikher and M. I. Shliomis, Adv. Chem. Phys. 87, 595 (1994).

[11] A. Morita and H. Watanabe, Adv. Chem. Phys. 56, 255 (1984).

[12] W. T. Coffey, J. L. Dejardin, Yu. P. Kalmykov, and S. V. Titov, Phys. Rev. E 54, 6462 (1996). 